\title{
The improved relative entropy for face recognition
}

\author{
Qi Rong ZHANG ${ }^{\text {a }}$, Ji Yan ZHANG and Yan WANG \\ Information Engineering, Guizhou University of Engineering Science, Bijie 551700, Guizhou, China
}

\begin{abstract}
The relative entropy is least sensitive to noise. In this paper, we propose the improved relative entropy for face recognition (IRE). The IRE method of recognition rate is far higher than the LDA, LPP method, by experimental results on CMU PIE face database and YALE B face database.
\end{abstract}

Keywords.Face recognition; Relative entropy; Improved relative entropy

\section{Introduction}

Through decades of development, face recognition has gradually get one of the most attract research domain in image analysis and computer vision, because a large number of applications. A large number of face recognition methods have been investigated such as Principal component analysis (PCA) [1], Linear discriminate analysis (LDA) [2], Independent component analysis (ICA) [3], Elastic bunch graph matching (EBGM) [4], Support Vector Machine (SVM) [5], Isometric feature mapping (ISOMAP) [6], Locally linear embedding (LLE) [7] [8], Laplacian eigenmap (LE) [9] [10], Locality Preserving Projections (LPP) [11]. Principal component analysis (PCA) [1], linear discriminant analysis (LDA) [2] and locality preserving projections (LPP) [11] are typical linear algorithms for face recognition. Sirovich and Kirby first applied PCA, and then the well-known Eigenface method is proposed. PCA and LDA intend to project the vectors which represent face images onto lower dimensional subspace. PCA does not work for the class information, so it's an unsupervised method. LDA can take full consideration of the class labels of the input data. So the LDA is should be a supervised method. In fact, LDA is to find the projection axes that when face image data points of different classes are projected as much as possible the far from, while face image data points of the same class are nearly as much as possible. LPP can construct a face subspace by preserving local information which is a recently appeared method. LPP is also an unsupervised method, but LPP is to maintain the human face image space of the local structure, while PCA is to maintain the global structure.

In statistical physics the most important concept is the Boltzmann's entropy [12]. This concept is introduced by Shannon into information theory, called Shannon's entropy [13]. Some scientific field research and application of the concept of entropy is a wide range of [14] [15] [16] [17] [18] [19] [20] [21]. Guang Deng found the relative entropy appears to be the least sensitive to noise through experiments [23]. However, Relative entropy has a drawback that it is potentially sensitive to noise in dark areas. Therefore, it is performing a pixelwise multiplication for an image to improve the relative entropy of this shortcoming. In this paper, we will use the improvement of the relative entropy for face recognition.

The rest part this paper is organized as follows: Section 2 introduced the proposed the improved relative entropy for face recognition (IRE). Section 3 reports the results of the experiment on CMU PIE face databases. Finally the conclusion of this paper is given in Section 4 .

\section{The relative entropy}

\subsection{The relative entropy}

\subsubsection{Shannon' entropy}

The Shannon' entropy can be defined as follows [13].

$$
\begin{array}{r}
H=H(P)=-\sum_{n=1}^{N} p_{*} \log _{2} p_{n} \\
p=\left\{p_{n}\right\}
\end{array}
$$

$p_{n}$ is the probability of occurrence of the ${ }^{i}$ th outcome or state.

\subsubsection{Relative entropy}

The relative entropy is defined as follows [22]

$$
D(Q \| P)=\sum_{n=0}^{N-1} q_{*} \log \frac{q_{*}}{p_{*}}(3)
$$

\footnotetext{
${ }^{\mathrm{a} Q i}$ Rong ZHANG: zqr_hk@163.com
} 


$$
\begin{array}{r}
=\frac{1}{N} \sum_{n=0}^{N-1}\left(\log A-\log x_{n}\right) \quad(4) \\
=\log \frac{A}{G}(5) \\
p_{n}=\frac{x_{n}}{S} \quad(6) \\
S=\sum_{*=0}^{N-1} x_{n}(7) \\
q_{n}=\frac{1}{N}(8) \\
A=\frac{S}{N}(9) \\
G=\exp \left(\frac{1}{N} \sum_{=-0}^{N-1} \log x_{n}\right) \quad(10)
\end{array}
$$

Where ${ }^{Q}$ is probability model, ${ }^{P}$ is also. $A$ is the arithmetic mean. ${ }^{G}$ is the geometric mean. So, Esq. (5) shows the relative entropy can be the ratio of the arithmetic mean to the geometric mean.

\section{The improved relative entropy for face recognition (IRE)}

The negative image is given as follows [23]

$$
y=M-x
$$

${ }^{x}$ is a pixel of the original image and $y$ is a pixel of negative image.

We can compute a pixel-wise multiplication as follows [23]

$$
Y=D_{1} \times D_{2}
$$

$D_{1}$ is the relative entropy of a pixel and $D_{2}$ is the relative entropy of a pixel .

The improved relative entropy for face recognition

Step.1. Input face images

Step.2. Compute the relative entropy of each pixel by Esq. (5), (9) and (10), using a neighboring (in this paper, the experiments using $3 \times 3$ neighborhood window).

Step.3. Computed by Esq. (11) and (12).

Step.4. Compute the Euclidean distance between of training face images and of testing face images.

$$
d\left(Y_{1}-Y_{2}\right)=\left\|Y_{1}-Y_{2}\right\|_{2}
$$

\section{Experiments}

\subsection{CMU PIE database}

We checked the feasibility and effectiveness of IRE method on CMU PIE face database.

The CMU PIE face database includes 68 persons with 41,368 face images with varied pose, illumination and expression which were photographed by thirteen synchronized cameras and twenty-first flashes in different pose, illumination and expression. In this experiment, we used twenty-one face images of sixty-eight persons with illumination variations. Each image is resized to $92 \times 112$. We arbitrarily selected face image from CMU face database. Fig.1. displayed face sample images from the CMU PIE database.

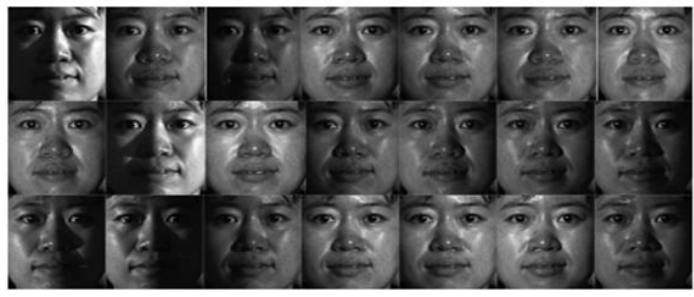

Fig.1 Illustration 21 images of one individual from CMU PIE face database.

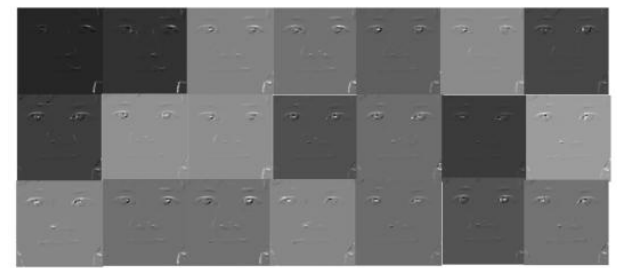

Fig.2 Illustration 21 images of one individual from CMU PIE face database by the relative entropy.

Table 1 displayed the three kinds of methods the highest recognition rate on the CMU PIE face database.

\begin{tabular}{llcl}
\hline Methods & 10 Train & 11 Train & 12 Train \\
\hline LDA & 91.34 & 92.17 & 94.14 \\
LPP & 72.38 & 75.64 & 77.05 \\
IRE & 93.32 & 94.22 & 95.66 \\
\hline
\end{tabular}

4.2 The YALE B database

On YALE B face database. We test positive images of 10 individuals who were shooting under 64 different lights. The above data set is divided into five subsets according to the direction of light: the best lighting conditions are Sub1, followed by Sub2, Sub5 worst.

We chose three images of each person as training samples Sub1, with the rest of the four samples for testing. Figure 3 displayed a sample image YALE B Sub1 face database.

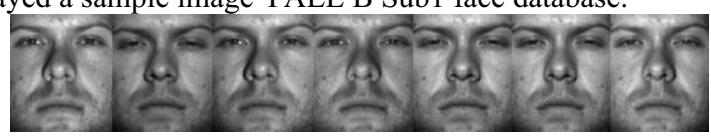

Fig.3 Illustration 7 images of one individual from YALE B subset 1 face database.

Table 2 displayed the three kinds of methods the highest recognition rate on the YALE B sub1 face database.

\begin{tabular}{ll}
\hline Methods & 3 Train \\
\hline LDA & 80.00 \\
LPP & 95.00 \\
IRE & 97.50 \\
\hline
\end{tabular}

We chose each person's six Sub2 image as the training sample, with the rest of the six samples for testing. Figure 4 displayed a sample image YALE B Sub2 face database.

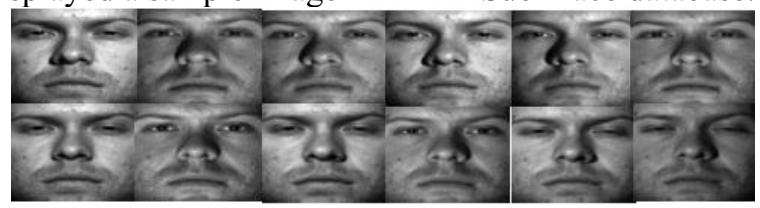

Fig.4Illustration 12 images of one individual from YALE B subset 2 face database. 
Table 3 displayed the three kinds of methods the highest recognition rate on the YALE B sub2 face database.

\begin{tabular}{ll}
\hline Methods & 6 Train \\
LDA & 81.67 \\
LPP & 91.67 \\
IRE & 96.67 \\
\hline
\end{tabular}

We chose each person's six Sub3 image as the training sample, with the rest of the six samples for testing. Figure 5 displayed a sample image YALE B Sub3 face database.

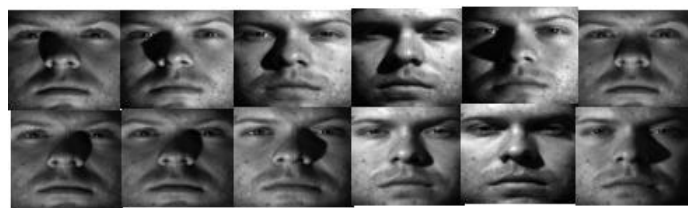

Fig.5 Illustration 12 images of one individual from YALE B subset 3 face database.

Table 4 displayed the three kinds of methods the highest recognition rate on the YALE B sub3 face database.

\begin{tabular}{ll}
\hline Methods & 6 Train \\
\hline LDA & 58.33 \\
LPP & 25.00 \\
IRE & 68.33 \\
\hline
\end{tabular}

We chose each person's seven Sub4 image as the training sample, with the rest of seven the samples for testing. Figure 6 displayed a sample image YALE B Sub4 face database.

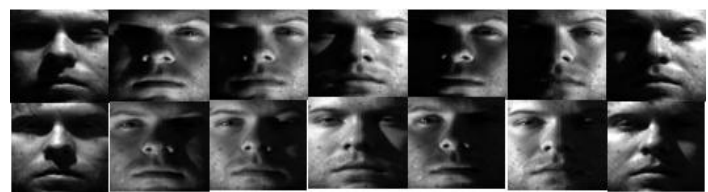

Fig.6 Illustration 14 images of one individual from YALE B subset 4 face database.

Table 5 displayed the three kinds of methods the highest recognition rate on the YALE B sub4 face database.

\begin{tabular}{lc}
\hline Methods & 7 Train \\
\hline LDA & 38.57 \\
LPP & 22.86 \\
IRE & 75.71 \\
\hline
\end{tabular}

\section{Conclusions}

In this paper, we proposed IRE method for face recognition. This method can make efficient recognition rate than LDA, LPP in CMU PIE face database and YALE B face database.

\section{Acknowledgments}

This work was supported by 2015 Annual Science and Technology Department of Guizhou Province, Bijie City Science and Technology Bureau, Guizhou University of Engineering Science Joint Science and Technology Fund Project, Cherry picking robot visionrecognition system research, Guizhou Branch of LH word [2015] No. 7599; the grants from 2014 Science and Technology Department of Guizhou Province, Bijie City Science and Technology Bureau, Bijie University of Science and Technology Joint Fund Program, Guizhou Branch of LH word [2014] No. 7536

\section{References}

1. M.Turk, A.P.Pentland, Eigenfaces for recognition, J. Cognitive Neurosci.3 (1) (1991)71-86.

2. P.N. Belhumeur, J. Hespanha, D.J. Kriegman, Eigenfaces vs. Fisherfaces: recognition using class specific linear projection, IEEE Trans. Pattern Anal. Mach. Intel. 20 (7) (1997) 711-720.

3. M. S. Bartlett, J. R. Movellan, T.J. Sejnowski, Face recognition by independent component analysis, IEEE Transactions on Neural Networks.13(6)(2002) 1450-1464.

4. L. Wiskott, J.M. Fellows, N. Kruger, C.V. Makburg, Face recognition by elastic bunch graph matching, IEEE Trans. Pattern Anal.Mach. Intell.19 (1997) 775-779.

5. G. Guo, S.Z. Li, K.L. Chan, Support vector machines for face recognition, Image and Vision Computing. 19 (9-10) (2001) 631-638.

6. J.B. Tenenbaum, V.de Silva, J.C. Langford, A global geometric framework for nonlinear dimensionality reduction, Science. 290 (5500) (2000) 2319-2323.

7. S.T. Roweis, L.K. Saul, Nonlinear dimensionality reduction by locally linear embedding, Science. 290 (5500) (2000) 23232326

8. L.K. Saul, S.T. Roweis, Think globally, fit locally: unsupervised learning of low dimensional manifolds, J.Mach. Learning. Res. (4) (2003) 119-155.

9. M. Belkin, P. Niyogi, Laplacian eigenmaps and spectral techniques for embedding and clustering, in: T.G. Dietterich, S. Becker, Z. Ghahramani(Eds.), Advances in Neural Information Processing Systems, vol.14, MIT Press, Cambridge, MA, USA, 2002, 585-591.

10. M. Belkin, P. Niyogi, Laplacian eigenmaps for dimensionality reduction and data representation, Neural Comput.15 (6) (2003)1373-1396.

11. X. He, S. Yan, Hu, P. Niyogi, H. Zhang, Face recognition using Laplacianfaces, IEEE Trans. Pattern Anal. Mach. Intell. 27 (3) (2005) 328340 . 
12. C.H.Liu, Nonsymmetric entropy and maximum nonsymmetric entropy principle, Chaos, Solitons \& Fractals. 40 (5) (2009) 2469-2474

13. C.E. Shannon, Bell Syst. Tech. J. 27 (1948) 379623.

14. Szwast Z, Sieniutycz S, Shiner JS. Complexity principle of extremality in evolution of living organisms by information-theoretic entropy. Chaos, Solitons \& Fractals.13 (9) (2002) 18711888.

15. Allegrini $\mathrm{P}$, Grigolini $\mathrm{P}$, Palatella $\mathrm{L}$. Intermittency and scale-free networks: a dynamical model for human language complexity. Chaos, Solitons \& Fractals. 20(1) (2004) 95-105.

16. Gontar V. Entropy principle of extremality as a driving force in the discrete dynamics of complex and living systems. Chaos, Solitons \& Fractals .11 (1-3) (2000) 231-236.

17. El Naschie MS. Dimensions and Cantor spectra. Chaos, Solitons \& Fractals.4 (11) (1994) 21212132.

18. El Naschie MS. On the topological ground state of E-infinity spacetime and the super string connection. Chaos, Solitons \&Fractals.32 (2) (2007) 468-70.

19. El Naschie MS. Superstrings, entropy and the elementary particles content of the standard model. Chaos, Solitons \& Fractals.29(1) (2006) 48-54

20. El Naschie MS. Internal Cantor distance and entropy of multidimensional Peano-Hilbert spaces. Chaos, Solitons \& Fractals.1993; 3:3618.

21. Tsallis C. Possible generalization of BoltzmannGibbs statistics. J Stat Phys.52 (1-2) (1988) 479487.

22. D.J.C. MacKay, Information Theory, Inference and Learning Algorithms. Cambridge, U.K. Cambridge Univ.Press, 2003

23. Guang Deng, An Entropy Interpretation of the Logarithmic Image Processing Model With Application to Contrast Enhancement, IEEE Transactions on Image Process.18 (5) (2009) 1135-1140. 